\title{
Adaptative Elimination of False Edges for First Order Detectors
}

\author{
Djemel ZIOU and Salvatore TABBONE
}

Département de mathématiques et d'informatique, université de Sherbrooke, Qc, Canada, J1K 2R1

Crin/Cnrs \& Inria-Lorraine, BP 239, 54506 Vandoeuvre-les-Nancy, France

\begin{abstract}
In this paper, we propose a new rule for the elimination of false edges produced by gradient detectors. This mule is based on the characteristics of the image and the properties of the detector used. The rule has been tested on realistic images using multi-scale edge detectors.
\end{abstract}

\section{Introduction}

In edge detection, the most commonly proposed schemes include three operations: differentiation, smoothing and labeling. Differentiation evaluates the derivatives of the intensity image. Smoothing consists in the reduction of noise included in the image. Labeling localizes edges and increases the signal-to-noise ratio by suppressing false edges. We are interested in this paper in the elimination of these false edges. We limit ourselves to gradient detectors. In this case false edges originate from noise. The commonly used classification rule of edges as being true or false is that the plausibility measure (i.e., the gradient modulus) of a true (false) edge is above (below) a given threshold. The threshold is the minimum acceptable gradient modulus. Due to the fluctuation of the gradient modulus, edges resulting from this binary decision rule are broken. So, this rule has been improved to take the continuity of edges into account. Indeed, the hysteresis algorithm uses two thresholds and a given edge is true if the gradient modulus of any edge point is above a low threshold and at least one is above a high threshold. Elsewhere, this edge is false.

Another aspect of the elimination of false edges concerns the threshold computation. Usually, a threshold is found using a trial-and-error process and the same threshold is used for all edges of an image. As we will show, the threshold is a function of edge characteristics and of the properties of the edge detector. Consequently, it is not easy to find a single value of threshold for a given image. We have proposed in $[4,7]$ a cleaning rule for multi-scale edge detection based on the behavior of the ideal step edge in scale space. Improvements for this algorithm are proposed in this paper to be used with any edge model and with a gradient operator combined with any smoothing filter. We have tested our algorithm with gradient and multi-scale detectors using various realistic images. In the next section, we show the influence of scale and edge orientation on the gradient modulus and on the gradient direction. By using the behavior of the gradient we propose two algorithms for the elimination of false edges in section 3. Finally, we discuss the results obtained. 


\section{Behavior of the Gradient}

In this section, we consider the influences of both the scale of the smoothing filter and the edge orientation on the gradient modulus and direction.

Let us consider the influence of the edge orientation on the gradient modulus and direction. For the sake of simplicity, we consider here only the case of an ideal step edge. This edge model is sufficient to show that it is suitable to take the edge orientation into account in the false edge elimination process. Consequently, the consideration of blurred edges and double edges does not affect our study. We have carried out this study in the continuous domain and shown that [6]: 1) The gradient magnitude of rotationally symmetric detectors is unaffected by edge orientation. For rotationally dependent detectors the gradient magnitude is often affected by edge orientation. However, there are rotationally dependent detectors whose gradient magnitude is unaffected by edge orientation as it is in the case of rotationally symmetric detectors. This influence is symmetric at edge orientation equal to $\pi / 4$ with an extremum at this orientation. 2) For rotationally symmetric detectors the estimated edge orientation is accurate; that is, the edge orientation $\theta$ is equal to the gradient direction $\psi$. For rotationally dependent detectors the estimation of edge orientation is often biased (i.e., $\theta \neq \psi$ ), even if the signal is noise free. However, there are rotationally dependent detectors such that $\theta=\psi$, as is the case for rotationally symmetric detectors. Furthermore, we have shown that in practice the property of invariance to rotation is not preserved, due to the tessellation of the image plane and the numerical error approximation. Rotationally dependent detectors are also influenced by these discretization problems and therefore remain the most sensitive to edge orientation.

Since the gradient modulus may be affected by edge orientation, the suppression of false edges may be sensitive to the orientation of the edge. In fact, the best threshold for one edge may be bad for another edge. We propose to compute an appropriate threshold for each edge pixel using the influence of the edge orientation (see next section).

Now, we will consider the influence of the scale on the edge plausibility (i.e., the gradient modulus). For the sake of simplicity, we examine the convolution of an one-dimensional smooth filter (normalized first order derivative) with one dimensional edges to infer the behavior of edges in two dimensions. The filter considered here is a tempered function fulfilling the regularization requirements of Tikhonov (i.e., exponential filter, Gaussian filter). Let us consider four different noise-free models of step edges which are frequently encountered in indoor images and which can be easily modeled: the ideal, the blurred, the pulse and the staircase model. The ideal edge usually corresponds to the clean border of the objects in the scene. The blurred edge comes from changes in illumination of the scene. The pulse and staircase edges result from mutual illumination between objects that are adjacent or from thin objects over a background. Although the problem of noise is not considered in our study, experimentation shows that the basic theory presented here is not affected by the presence of noise. The influence of the scale on the estimated contrast has been studied in $[4,5,7]$ and is sum- 
marized by: 1 ) ideal edge: the plausibility is independent of the scale; 2 ) blurred edge: the plausibility is an increasing function of the scale. When the scale is large it is equal to the edge contrast; 3 ) pulse edge: the plausibility decreases when the scale increases. For a large scale, the weaker edge disappears while the stronger one remains, its plausibility is equal to the difference of the two steps; 4) staircase edge: the plausibility of the two maxima increases with the scale. At large scale, the plausibility is equal to the sum of the two steps.

Except the ideal edge, the plausibility of edges increases or decreases with the scale. For a blurred edge, the plausibility is very low at fine scale and it is difficult to distinguish it from noisy edges using only the contrast as criterion. This is also true for a pulse edge at high scale. One practical solution for this problem is to associate to each edge point its own threshold and to bind the scale used. Figure 1.a shows a grey level image containing a pulse edge and a blurred edge. To this image, we have added a strong Gaussian white noise with variance equal to 225 . The contrast of the pulse edge is $(50,20)$ and the contrast of the blurred edge is 25 and its width is 6 . Figure 1.b presents edges obtained using Canny's detector [1] at scale $\sigma=1.5$. The gradient modulus of the pulse is $(41,8.25)$ and the gradient modulus of the blurred is 17.5. Although the image has been smoothed, the amplitude of noise is greater than both the gradient modulus of the weak edge of the pulse and the gradient modulus of the blurred. Therefore, by using a single threshold it is difficult to suppress false edges while preserving true edges (see figure 1.c). It is necessary to use a specific threshold for each of those edges.

\section{Elimination of False Edges}

We have shown in the previous section that if a cleaning algorithm is used, the choice of the threshold must take into account the behavior of edges in scale space and the sensitivity of the plausibility measure to the edge orientation. This means that it is suitable to compute one threshold per pixel and therefore to associate for each edge model a specific computation rule. Given an edge model $m(x, y)$, thresholds are computed according to this rule:

$$
t(x, y)=t_{0} c_{m, \theta, s}(x, y)
$$

$t_{0}$ is a given threshold which can be considered as the smallest acceptable contrast at a given scale $s$. The same value of $t_{0}$ is used for all edge models. There is no known rule which can be successfully used for choosing this threshold. The trial-and-error process remains a reliable means for choosing the appropriate $t_{0}$. The quantity $c_{m, \theta, s}$ (see eq. 2) represents the sensitivity of the detector to the edge orientation $(\theta)$ and the influence of the scale $(s)$. The gradient modulus of the response of the filter $f(x, y, s)$ describes the influence of the scale and of the edge orientation. Since $c_{m, \theta, s}$ must be independent of the contrast, we define it as the ratio of the gradient modulus of the response of the filter $f(x, y, s)$ to the gradient modulus of the response of the filter $f(X, Y, s)$ :

$$
c_{m, \theta, s}=\sqrt{\frac{\left(I(x, y) * f_{x}^{\prime}(x, y, s)\right)^{2}+\left(I(x, y) * f_{y}^{\prime}(x, y, s)\right)^{2}}{\left(I(x, y) * f_{x}^{\prime}(X, Y, s)\right)^{2}+\left(I(x, y) * f_{y}^{\prime}(X, Y, s)\right)^{2}}}
$$


where $s$ is the scale used, $X=x \cos (\theta)+y \sin (\theta), Y=-x \sin (\theta)+y \cos (\theta)$, $f(X, Y, s)$ is the rotation an angle $\theta$ about the origin of the filter $f(x, y, s)$. The quantity $\theta$ is the estimated edge orientation which approximates the gradient direction $\psi$. The gradient modulus of the response of $f(X, Y, s)$ (i.e.; the denominator in eq. 2) is orientation independent. In fact, let $x=\rho \cos (\phi)$ and $y=\rho \sin (\phi)$ be the polar coordinate system, $m(X)$ a linear edge of any type with an orientation $\theta \in[0, \pi / 2]$ and $f(x, y, s)$ a smoothing filter. It is easy to show that:

$$
\int_{0}^{+\infty} \int_{0}^{2 \pi} f(\rho \cos (\phi-\theta), \rho \sin (\phi-\theta)) m(\rho \cos (\phi-\theta))-f(\rho, \phi) m(\rho \cos (\phi)) \rho d \rho d \phi=0
$$

This result means that the convolution of an edge with orientation $\theta$ with a filter $f(x, y, s)$ taken in the direction $\theta$ is equivalent to the convolution of the filter taken in the horizontal direction with a vertical edge. As we have shown earlier, in this case, the value of the denominator in (eq. 2) is the largest or the smallest depending on the detector used. Therefore, the value of $c_{m, \theta, s}(x, y)$ is one if the gradient modulus is orientation independent as is the case with Canny's detector. Elsewhere, it can be smaller (greater) than one when the gradient modulus decreases (increases) depending on the edge orientation and the detector used. For instance, for Deriche's detector[3] $c_{m, \theta, s}(x, y)$ is greater than one. The convolution of the image and the filter $f(X, Y, s)$ can be computed immediately using convolution masks. The cleaning algorithm can be summarized as follow: given the first order derivatives of the smoothed image $I(x, y) * f_{x}^{\prime}(x, y, s), I(x, y) *$ $f_{y}^{\prime}(x, y, s)$, for each edge pixel compute $I(x, y) * f_{x}^{\prime}(X, Y, s), I(x, y) * f_{y}^{\prime}(X, Y, s)$ and $c_{m, \theta, s}$ (eq. 2) and then compute $t$ (eq. 1).

In a multi-scale edge detection scheme, one needs to suppress false edges in all recovered images by cleaning each of them before the edge combination process. In general, this method is tedious since the trial-and-error process is required at each scale. To automate this task, we have proposed in $[4,7]$ an algorithm based on threshold propagation. More precisely, it is possible to compute a threshold for given edge pixels obtained at a high scale and to propagate it automatically at lower scales. The choice of the high scale as a starting point is based on the fact that, at this scale, there are few false edges and they are usually easy to suppress. We give here some improvements of this algorithm taking into account the effect of edge orientation and extending it to any edge model and to any edge detector. The multi-thresholding algorithm is given as follows:

1. Threshold edge pixels obtained at a high scale using the algorithm mentioned above; that is $t_{h}(x, y)=t_{0} c_{m, \theta, s_{h}}(x, y) . t_{0}$ represents the smallest acceptable contrast of horizontal and vertical edges at high scale. $c_{m, \theta, s_{h}}$ used in this case represents the sensitivity of the detector to the edge orientation at the same scale.

$$
c_{m, \theta, s_{h}}(x, y)=\sqrt{\frac{\left(I(x, y) * f_{x}^{\prime}\left(x, y, s_{h}\right)\right)^{2}+\left(I(x, y) * f_{y}^{\prime}\left(x, y, s_{h}\right)\right)^{2}}{\left(I(x, y) * f_{x}^{\prime}\left(X, Y, s_{h}\right)\right)^{2}+\left(I(x, y) * f_{y}^{\prime}\left(X, Y, s_{h}\right)\right)^{2}}}
$$

2. Threshold edges obtained at smaller scales. The threshold is computed automatically from the one obtained at a high scale; that is, $t(x, y)=$ 


$$
\begin{aligned}
& t_{h}(x, y) c_{m, s_{h}, s}(x, y) \\
& c_{m, s_{h}, s}(x, y)=\sqrt{\frac{\left(I(x, y) * f_{x}^{\prime}(X, Y, s)\right)^{2}+\left(I(x, y) * f_{y}^{\prime}(X, Y, s)\right)^{2}}{\left(I(x, y) * f_{x}^{\prime}\left(X, Y, s_{h}\right)\right)^{2}+\left(I(x, y) * f_{y}^{\prime}\left(X, Y, s_{h}\right)\right)^{2}}}
\end{aligned}
$$

The influence of the orientation is considered only at the high scale. This influence is transcribed in $t_{h}$ and therefore it is propagated to the low scales.

\section{Experimental Results and Conclusion}

We have experimented our algorithm using various real images and various edge detectors. We present here results of Deriche's detector. It should be noted that Deriche's detector is not rotationally symmetric. Consequently, edges produced by this detector are not easy to classify. To eliminate false edges we have used only one threshold combined to the binary decision rule. The use of the hysteresis algorithm leads to better results. Figure 2.a presents an indoor image which contains various edge models. Figures $2 . b, 2 . c$ and $2 . d$ are respectively edges obtained at scale $\alpha=0.9$, true edges and false edges $\left(t_{0}=9\right)$. Figure 2.e shows edges obtained at $\alpha=1.2$. At this scale, false edges are suppressed by the propagation of the threshold used at $\alpha=0.9$. Figures $2 . f$ and $2 . g$ display respectively true edges and false edges.

We have shown that the use of the same threshold for all edges in the image does not lead to sufficient results. Our method takes into account the following parameters: edge orientation, signal-to-noise ratio, edge model, behavior of edges in scale space, multi-scale processing, properties of both the smoothing filter and the differentiation operator. Experiments show the efficiency of the classification scheme to distinguish false edges from true ones and for the propagation of thresholds between scales.

\section{References}

1. J.F. Canny. A Computational Approach to Edge Detection. IEEE Trans. on (PAMI), 8(6):679-698, 1986.

2. J.J. Clark. Authenticating Edges Produced by Zero-Crossing Algorithms. IEEE Trans. on (PAMI), 11(1):43-57, 1989.

3. R. Deriche. Using Canny's Criteria to Derive a Recursive Implemented Optimal Edge Detector. Int. Jour. Comp. Vision, 1(2):167-187, 1987.

4. S. Tabbone. Edge Detection, Subpixel and Junctions Using Multiple Scales. PhD thesis, Institut National Polytechnique de Lorraine, France, (In french), 1994.

5. D.J. Williams and M. Shah. Edge Characterization Using Normalized Edge Detector. CVGIP, 55:311-318, 1993.

6. D. Ziou. Edge Contrast and Orientation Estimation: What About Rotation Invariance? IEEE Trans. on (PAMI), Submitted, 1994.

7. D. Ziou and S. Tabbone. A Multi-Scale Edge Detector. Pattern Recognition, 26(9):1305-1314, 1993. 

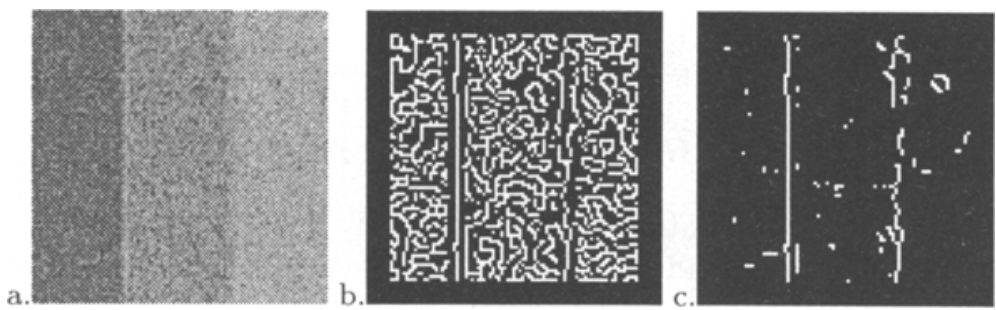

Fig. 1. a) Original image: $99 \times 99$ of 170 grey levels, left edge is a pulse of a contrast $(50,20)$, right edge is a blurred of contrast 25 and width 6 pixels, the variance of noise is 255. b) Edges obtained using Canny's detector with $\sigma=1.5$. c) Cleaned edges, the threshold used is 15 .
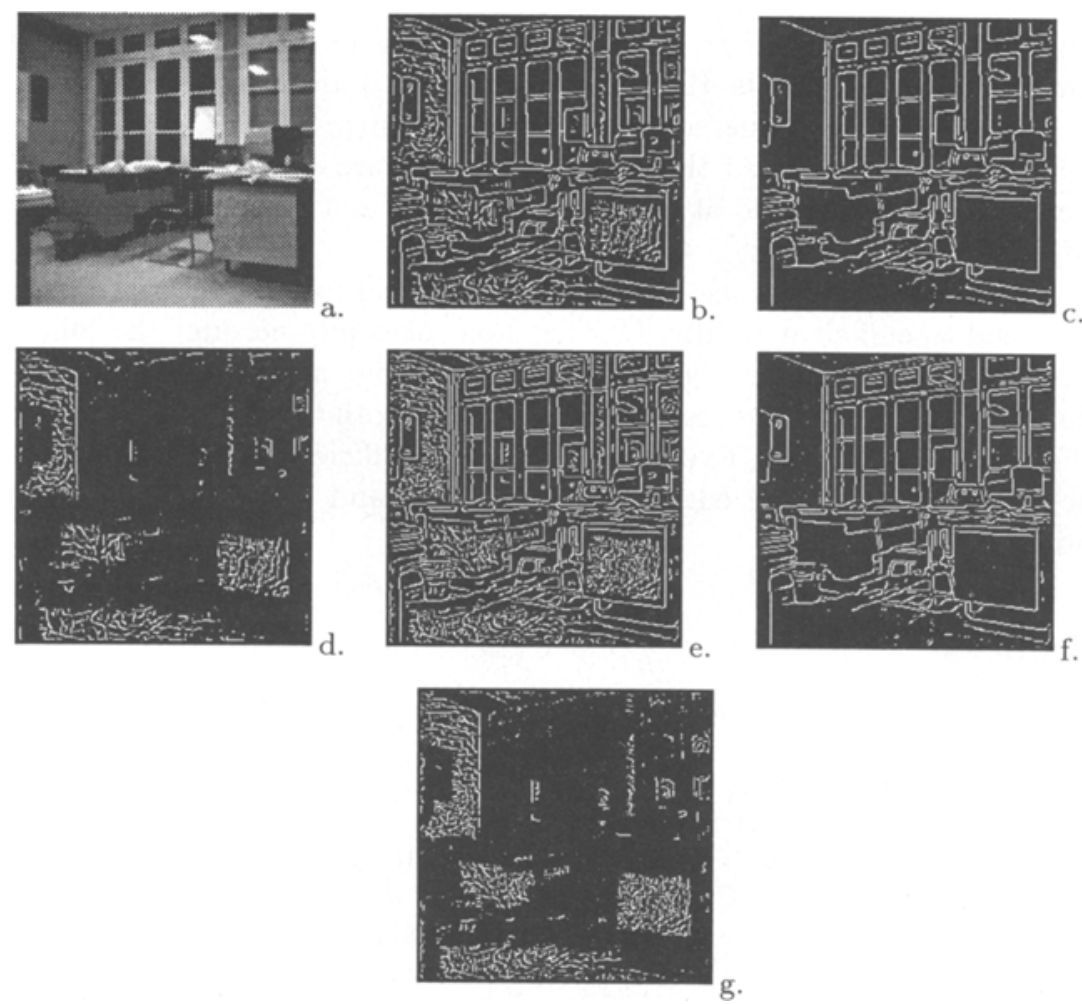

Fig. 2. (a) Indoor image $(256 \times 256$ pixels and 256 grey levels $)$. (b) Edges resulting from Deriche's detector at the scale $\alpha=0.9$. (c) and (d) True edges and noisy edges $\left(t_{0}=9\right)$. (e) Edges obtained at $\alpha=1.2$. ( $\left.f\right)$ and $(g)$ True edges and noisy edges resulting from the procedure of threshold propagation. 